\title{
Kliniken Nordoberpfalz und RECOM-GRIPS: Eine 10-jährige Erfolgsgeschichte
}

Seit 2008 arbeitet man am Hauptstandort in Weiden mit RECOMs Patientenakte. Ausschlaggebend für die Entscheidung zur Digitalisierung waren steigende Dokumentationsanforderungen wegen gesetzlicher Vorgaben und höheren Pflegeaufwandes. Wichtiger noch: die Steigerung der Pflegequalität.

In etwa 90 Prozent aller deutschen Krankenhäuser werden Heil- und Pflegemaßnahmen noch auf Papier dokumentiert: Hoher Aufwand, Fehleranfälligkeit und unerwünschte Doppeldokumentation sind die Folge, oft kommt es am Ende auch zu Abrechnungsproblemen mit den Krankenkassen. Das Ziel im Sinne der Patienten: eine lückenlose, fehlerfreie Dokumentation, die gleichzeitig als Basis für rechtssichere Abrechnungen gegenüber den Krankenkassen dient.

Bereits im Rahmen des Studiums kam der heutige Pflegedirektor der Kliniken Nordoberpfalz, Thomas Baldauf, mit verschiedenen Lösungen zur elektronischen Pflegedokumentation in Berührung. Im Jahr 2007 reifte der Entschluss, eine EDVgestützte Pflegeplanung und -dokumentation einzuführen. Da mit Siemens Medico (heute Cerner) bereits ein Krankenhausinformationssystem (KIS) im Einsatz war, wurde analysiert, inwieweit sich die hohen Anforderungen erfüllen lassen bzw. ob es mit der Implementierung einer weiteren EDV-Lösung zu unerwünschten Redundanzen käme.

Die Entscheidung für die digitale Patientenakte (ePA) RECOM-GRIPS fiel aus zwei Gründen: Die Lösung basiert auf ENP als Pflegesprache und Praxisleitlinie, zudem überzeugt der Funktionsumfang. RECOM bindet die Anwender bei der Handhabung eng mit ein, liefert umfassende pflegewissenschaftliche Inhalte und ermöglicht eine sehr differenzierte Beschreibung des Patientenfalles. „Den Ausschlag hat schlussendlich die hohe pflegewissen- schaftliche Expertise der Firma RECOM mit eigenem Team aus Pflegewissenschaftlern gegeben“, erinnert sich Michaela Sauer, die das Projekt von an Anfang an begleitete. Die Krankenschwester arbeitete auf der Pilotstation, bevor sie mit der Leitung des ePA-Projektes beauftragt wurde. Ebenso wichtig war die nahtlose Interoperabilität mit Cerner Medico. „Die Best-ofBreed-Strategie geht nur auf, wenn die einzelnen Systeme perfekt harmonieren“,

Kliniken Nordoberpfalz: Stark in der Region

Mit über 100000 Patienten pro Jahr und rund 3000 Angestellten ist die Kliniken Nordoberpfalz AG leistungsfähiger Gesundheitsdienstleister und gleichzeitig größter Arbeitgeber der Region. Zu dem GesundheitsUnternehmen zählen sechs AkutKrankenhäuser, zwei Rehabilitationseinrichtungen, ein Aus-, Fort- und Weiterbildungszentrum, eine Pflegeeinrichtung und drei medizinische Versorgungszentren.

Neben RECOM-GRIPS setzen das Klinikum Weiden sowie alle angeschlossenen Häuser seit vielen Jahren auch die Patientenaufklärungssoftware E-ConsentPro Classic von Thieme Compliance ein. erklärt Robert Dworschak. „Da Cerner die entsprechende Medico-Schnittstelle bereitstellte, klappte die Co-Existenz der beiden Systeme einwandfrei“, ergänzt der Leiter der Zentralen EDV-Abteilung am Standort Weiden.

\section{Pilotstation und Rollout ohne große Stolpersteine}

Bereits Ende des ersten Quartals 2008 wurde eine unfallchirurgische-orthopädische Pilotstation eingerichtet. Bevor GRIPS in den Live-Betrieb ging, wurde das 17-köpfige Pflegepersonal intensiv geschult. „Die parallele Nutzung der bisher handschriftlichen Dokumentation und der neuen digitalen Lösung wäre einfach zu arbeitsintensiv und zu fehleranfällig gewesen“, erinnert sich Dworschak. „Wir haben von Anfang an mit einem klaren Schnitt geplant", so der EDV-Leiter. „Die Kolleginnen und Kollegen auf der Pilotstation wurden auch nach der Umstellung auf GRIPS intensiv betreut und angeleitet“, berichtet Sauer, heute ePA-Beauftragte bei den Kliniken Nordoberpfalz. Hierfür waren keine externen Trainierstunden erforderlich. Der Schulungsaufwand lässt sich mit den inhouse vorhandenen Ressourcen stemmen, ohne zusätzliche Kosten zu verursachen. Bereits beim Piloten kam ein mobiler Computerwagen mit autarker Stromversorgung zum Einsatz. Voraussetzung hierfür war der Ausbau der WLAN-Infrastruktur, die heute im gesamten Haus flächendeckend sichergestellt ist. Nach dem erfolgreichen Pilotprojekt erfolgte zeitnah der Rollout am Stammhaus in Weiden. 


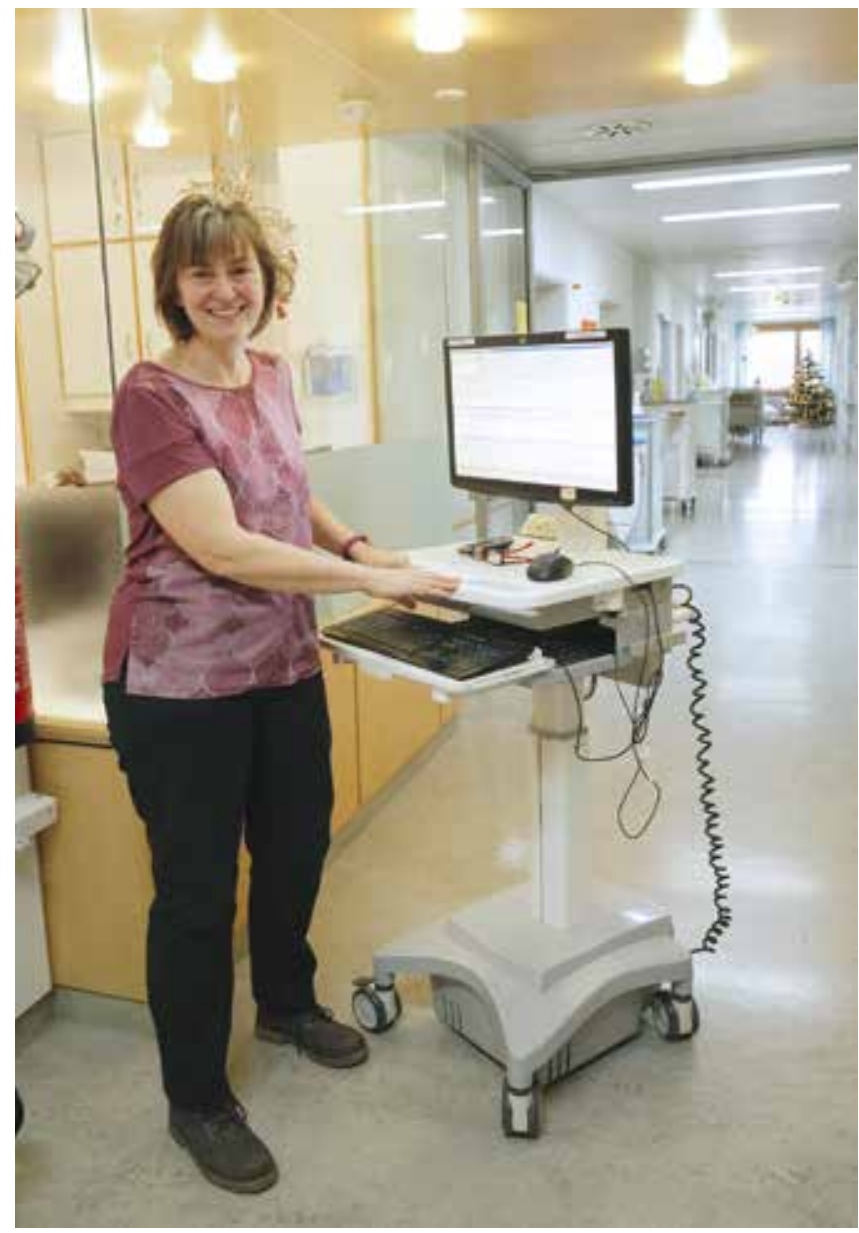

Abgebildete Person: Michaela Sauer (Teamleitung ePa, Kliniken Nordoberpfalz AG, Klinikum Weiden). Foto: RECOM $\mathrm{GmbH}$

\section{Aus dem Pflegealltag nicht mehr wegzudenken}

Die technische Betreuung und Schulung der Mitarbeiter rund um die Themen ePA und PKMS (PflegekomplexmaßnahmenScore) übernimmt heute ein fünfköpfiges Team unter Leitung von Sauer. Aus der anfänglichen Hersteller-Kunden-Beziehung zwischen RECOM und dem Klinikum ist über die Jahre eine Partnerschaft auf Augenhöhe erwachsen. „Auch bei der Weiterentwicklung der Lösung arbeitet RECOM eng mit uns zusammen und berücksichtigt unsere Wünsche“, erklärt Sauer. „Wir werden zudem regelmäßig mit neuen Inhalten und Funktionen versorgt." So kann der Klinikverbund darauf vertrauen, auf dem aktuellen Stand pflegewissenschaftlicher und berufspolitischer Anforderungen zu sein.

Entscheidend für das Krankenhaus war auch, dass nicht nur die Software regelmäßig aktualisiert wird, sondern auch pflegerische Inhalte (European Nursing care Pathways, ENP) auf wissenschaftlichem Niveau und stets auf aktuellstem Stand sind. So lassen sich vorhandene Ressourcen ganz auf die Pflege ausrichten.

Die digitale Patientenakte ist zu einem selbstverständlichen, akzeptierten und unverzichtbaren Instrument im Klinikalltag geworden. Kommen neue Mitarbeiter hinzu, profitieren sie vom hohen Standardisierungsgrad und der pflegewissenschaftlichen Sprache. Übertragungsfehler lassen sich weitgehend ausschließen, was gerade bei der Medikation einen enormen Sicherheitszuwachs bedeutet. Insgesamt hat sich die Dokumentationsqualität deutlich verbessert. Derzeit ist die Software im Klinikum Weiden auf den Akutstationen im Einsatz, ebenso im Krankenhaus Kemnath. Weitere sind in Planung.

\section{Reizthema PKMS wird stark entschärft}

Wichtige Verbesserungen bringt GRIPS auch für die PKMS-Beauftragten im Klinikverbund. Aufgrund der einheitlichen, strukturierten und stets lesbaren Dokumentation fällt die Betreuung der Stationen erheblich leichter. Die vorhandene Dokumentation lässt sich im Vorfeld einer Prüfung jederzeit berufsgruppenübergreifend einsehen. Die Rechtssicherheit der Dokumentation ist dank automatischer Hinterlegung von Datum, Uhrzeit und dokumentierender Person deutlich höher als bei handschriftlicher Vorgehensweise. Während in vielen Einrichtungen die PKMS-Dokumentation parallel zur Regeldokumentation erfolgt, verhindert GRIPS zuverlässig diesen Mehraufwand. PKMS-Gründe und PKMS-Score werden automatisch aus der Regeldokumentation ausgeleitet. Dank der nachvollziehbaren Score-Ermittlung verlaufen MDK-Prüfungen inzwischen wesentlich schneller und einfacher als vor Einführung der elektronischen Patientenakte. Die Quote der positiv begutachteten PKMS-Fälle ist bei den Kliniken Nordoberpfalz auf über 90 Prozent angestiegen. Michaela Sauer hat noch einen entscheidenden Tipp: „Binden Sie von Anfang an alle beteiligten Berufsgruppen in das Projekt mit ein. Denn im Nachhinein fällt es deutlich schwerer, einzelne Personen von den Vorteilen der Patientenakte zu überzeugen.“

\section{Patrick Afschar Kaboli}

Journalist 